\title{
Cardiomyopathies
}

The national society journals present selected research that has driven recent advances in clinical cardiology

\section{Perry M Elliott ${ }^{1}$, Saidi A Mohiddin ${ }^{2}$}

${ }^{1}$ The Heart Hospital, 16-18 Westmoreland Street, London; UK, '2Department of Cardiology, The London Chest Hospital, London, UK

The article was first published in Heart (Heart 2011;97 (23):1914-1919, doi: 10.1136/heartjnl-2011-301266) and in republished with permission.

\section{Introduction}

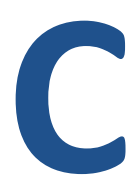

ardiomyopathies are myocardial disorders in which the heart muscle is structurally and functionally abnormal in the absence of coronary artery disease, hypertension, valvular disease and congenital heart disease, sufficient to cause the observed myocardial abnormality. They are classified into a number of morphological and functional phenotypes that can be caused by genetic and non-genetic mechanisms. A few key themes have been dominant in 201011 , foremost of which are the use (and interpretation) of increasingly sophisticated genetic analyses and the use of new non-invasive imaging techniques to study clinical phenotypes. There were few advances in treatment reported and it remains clear that there is a need for properly conducted randomised trials in all forms of cardiomyopathy.

\section{Hypertrophic cardiomyopathy (HCM)}

$\mathrm{HCM}$ is defined by the presence of myocardial hypertrophy unexplained by loading conditions. It is a genetic disorder predominantly caused by mutations in sarcomere protein genes, but other genetic diseases, including metabolic disorders such as Anderson-Fabry disease, account for a substantial minority of cases. ${ }^{1}$

The literature over the past year illustrates the continued importance of conventional diagnostic tools such as ECG and echocardiography in the diagnosis of HCM, but various refinements using different technical approaches, such as deformation imaging and 3D echo, were reported. Perhaps the most important advance has been the use of cardiac MRI. Two aspects were prominent: the ability of cardiomagnetic resonance (CMR) to detect myocardial segments 'invisible' to echocardiography (eg, posterior septum and apex) and probably more importantly, the ability to image myocardial scar using gadolinium enhancement. Numerous papers have examined the pattern and distribution of scar and its relation to clinical presentation and prognosis. ${ }^{2-4}$ Most data suggest that the presence of scar is predictive of heart failure rather than sudden cardiac death, but larger unbiased cohort studies are required. Methods to detect diffuse fibrosis are likely to be even more important as this probably develops at an early stage of the disease and represents an important therapeutic target. ${ }^{56}$

\section{Management}

Many patients with HCM experience premature death or have decades of poor health. The current stateof-the art in the management of each patient with HCM focuses on three aspects of the disease: identification of individuals who are at increased risk of sudden cardiac death and thus might benefit from implantable cardioverter-defibrillator (ICD) treatment; relief of left ventricular (LV) outflow tract obstruction and palliation of limiting symptoms caused by systolic or diastolic dysfunction. In all three areas, treatments remain suboptimal, particularly in the prevention of progressive heart failure. Potential benefits of presymptomatic diagnoses in affected family members, largely justified on the basis of sudden death prevention, is also an emerging theme in many papers. ${ }^{78}$

\section{Relief of LV outflow obstruction}

Several meta-analyses comparing the results of septal myectomy with septal alcohol ablation have been published. ${ }^{9-12}$ In general, these studies show that alcohol septal ablation is associated with broadly similar mortality rates and improvements in functional status to those reported for surgical treatment, albeit with a higher risk of permanent pacemaker implantation and greater postintervention outflow tract gradient. Some series have shown an excess of deaths after alcohol ablation, resulting in cautionary comments about the safety of this procedure. ${ }^{10}$ The search for alternative treatments for outflow tract obstruction also continues with reports of radiofrequency ablation of the septum and reappraisal of dual chamber atrioventricular sequential pacing..$^{13-15}$ In the absence of a randomised comparison, the controversies about the relative strengths and weaknesses of each of these treatments will continue. Currently, invasive treatment of LV outflow obstruction is recommended only in patients with drug-refractory symptoms. 


\section{Sudden cardiac death}

In contemporary practice, a small number of clinical risk markers are used in aggregate to predict the probability of sudden cardiac death and the need for ICDs. ${ }^{16}$ ${ }^{17}$ While remaining valid, current approaches have important limitations. In particular, many patients receiving ICDs for primary prevention never require device intervention, are exposed to risks of device-related complications and remain at risk of premature death from thromboembolic stroke and progressive heart failure. ${ }^{18}$ In addition, while the genetic causes of HCM in children are similar to those in adults, ${ }^{19}$ conventional risk prediction algorithms may not apply to paediatric populations. ${ }^{20}$ Further data on risk prediction and randomised trials of interventions that might prevent disease progression are clearly necessary.

Refractory symptomatic HCM

It has been hypothesised that excessive sarcomeric energy consumption is important in the pathophysiology of HCM and other heart muscle diseases. The mechanism of this energetic disturbance is not understood, but high energy phosphate ratios are reduced in patients with mutations in sarcomere proteins and little or no hypertrophy, suggesting that energy deficiency is a fundamental characteristic of the HCM phenotype. As disturbance of fatty acid metabolism is one the key drivers of inefficient energy use in the failing heart, ${ }^{21}$ perhexiline, an inhibitor of mitochondrial fatty acid uptake, was used to treat exertional symptoms in a randomised placebo-controlled trial. Perhexiline improved symptoms, exercise capacity and diastolic function during exercise in symptomatic patients with non-obstructive HCM, suggesting that this and similar drugs might be of use in some patients ${ }^{22}$

\section{Family screening}

Recently published cost-benefit analyses comparing genetic and clinical screening strategies provide an economic argument for the use of genetic testing in family screening. ${ }^{78}$ However, the published models are based on the assumption that risk-prediction algorithms developed and validated in populations at relatively high risk (largely comprising proband patients) apply equally to cohorts at lower baseline risk (Moons et al., unpublished data). In addition, confounders such as family size, disease penetrance, genetic variants of uncertain significance and the relatively high frequency of compound heterozygosity are largely unaccounted for in current cost-efficacy models. Prospective evaluation of different screening strategies is necessary.

Arrhythmogenic right ventricular cardiomyopathy Arrhythmogenic right ventricular cardiomyopathy (ARVC) is a genetic heart muscle disease characterised histologically by cardiomyocyte loss and replacement by fibrous or fibrofatty tissue, and clinically by arrhythmia, sudden cardiac death and heart failure. In many people, the disease is caused by mutations in genes that encode components of the intercalated disc of cardiomyocytes. ${ }^{23}$ Clinically, ARVC is difficult to diagnose, requiring integration of data from family members, genetic test- ing, electrocardiography and imaging techniques. ${ }^{24}$ The major management problems in ARVC are prevention of sudden cardiac death and treatment of symptomatic arrhythmia and heart failure.

\section{Aetiology}

Systematic family studies have shown that ARVC is inherited in up to $50 \%$ of cases as an autosomal dominant trait with incomplete penetrance and variable clinical expression. To date, most studies have shown that the majority of familial cases are caused by heterozygous mutations in genes encoding desmosomal proteins, but other genes have been implicated including transforming growth factor $\beta 3$ and transmembrane protein 43 (TMEM43), a cytoplasmic membrane protein. ${ }^{23}$ ${ }^{25}$ Over the past year, further evidence of genetic heterogeneity has been demonstrated by the discovery of pathogenic mutations in desmin, an intermediate filament protein, and titin. ${ }^{26-28}$ In addition, studies continue to report complex genetic status in many patients with multiple variants in different desmosomal genes. ${ }^{29}$ The presence of multiple mutations appears to increase the severity of the clinical phenotype, but also poses a challenge for the interpretation of genetic testing, particularly with regard to variants that may occur in normal populations which do not cause disease in themselves, but might conceivably alter disease susceptibility in the presence of other genetic or environmental factors.

\section{Diagnostic criteria}

As in other heart muscle diseases, current diagnostic paradigms for ARVC represent the latest iteration of a scientific and clinical narrative that began with the study of severe cases presenting with sudden cardiac death or ventricular arrhythmia. Pathological examination of postmortem specimens from these advanced forms of the disease were used to establish histological hallmarks of the disease-namely, fibrofatty replacement, aneurysm formation and right ventricular dilatation. Thereafter, standard clinical tools such as ECG, ventriculography, echocardiography and latterly cardiac MRI have been used to diagnose ever more subtle manifestations of this histological phenotype. Recognition that the disease is a familial trait caused by mutations in genes that code for proteins of the intercalated disc, has added to this complexity. ${ }^{30} \mathrm{~A}$ rationalisation of these different aspects of the disease formed the basis for modified diagnostic criteria published simultaneously in Circulation and the European Heart Journal in 2010.24 This important paper has already defined future clinical and scientific enquiry, and evidence from families suggests that the sensitivity and specificity of the criteria have improved. ${ }^{31} 32$ Nevertheless, the greater emphasis on quantification and genotyping is already posing significant diagnostic and management challenges. For example, athletic training alone may result in a phenotype that fulfils criteria in the absence of genetic evidence for the disease ${ }^{33}$ and ECG criteria may also show considerable variability with time. ${ }^{34}$ 


\section{Prevention of sudden death}

In spite of the reputation of ARVC as a major cause of sudden cardiac death, prospective data on the risk of sudden death in unselected populations and its prevention are surprisingly few. Current AHA/ACC/ESC 2006 guidelines for the management of patients with ventricular arrhythmias and the prevention of sudden cardiac death recommend ICD implantation in patients with ARVC who have documented sustained ventricular tachycardia (VT) or ventricular fibrillation (VF) and who are receiving optimal medical treatment with reasonable expectation of survival with a good functional status for more than 1 year. Recommendations in patients without these features are necessarily more speculative. Retrospective analyses have identified a number of possible predictors of adverse outcome in probands, including early age of onset of symptoms, participation in competitive sports, a malignant family history, severe right ventricular dilatation, LV involvement, syncope, episodes of ventricular arrhythmias and increased QRS dispersion on 12-lead ECG. In 2010, Corrado and colleagues published a study on 106 consecutive patients with ARVC who received an ICD based on one or more arrhythmic risk factors, such as syncope, non-sustained VT, familial sudden death and inducibility at programmed ventricular stimulation. ${ }^{35}$ During follow-up, $24 \%$ of patients had appropriate ICD interventions, 17 of which (16\%) were for VF or ventricular flutter. All patients survived to 48 months. Syncope was the most important predictor of ICD intervention but programmed ventricular stimulation had a low accuracy for predicting ICD treatment. These data add to current advice for ICD implantation in symptomatic patients, but the issue of primary prevention in asymptomatic patients remains a question for the future.

\section{Dilated cardiomyopathy (DCM)}

DCM, when defined as LV dilatation and systolic impairment in the absence of previous myocardial infarction, is one of the commonest heart muscle diseases in developed countries.

Over the past year, research emphasising the importance of genetics in the aetiology of inherited and apparently acquired forms of DCM has been a prominent feature. Tissue characterisation imaging with CMR is another notable feature of recent DCM research, with studies suggesting that it provides additional diagnostic and prognostic information. Patient management continues to consist largely of standard symptomatic and prognostic heart failure treatments, but recent work has begun to identify the importance of aetiology in determining management.

\section{Aetiology}

The difficulty in distinguishing between inherited and acquired cases of DCM remains a major challenge as the profile of clinical findings rarely helps to identify aetiology. In cases of sporadic disease (ie, in the absence of affected family members), circumstantial evidence may suggest the causative cardiac injury is inflammatory, toxic, load or heart rate dependent, or due to metabolic abnormalities. However, recently published data suggest that genetic susceptibility is often underestimated in apparently sporadic disease. In the past, animal data have demonstrated the importance of host genetic factors in determining susceptibility to cardiomyotrophic viral pathogens ${ }^{36}$; in 2010 , an association between myocarditis and common gene variants was reported for the first time in humans. ${ }^{37}$ In peripartum cardiomyopathy, studies demonstrated an association of a chromosomal locus with peripartum $\mathrm{DCM}^{38}$ and the presence of undiagnosed DCM in first-degree family members (3 of 10) of women diagnosed with peripartum DCM. ${ }^{39}$

Recent genetic studies have also challenged previously accepted concepts of disease pathogenesis. ${ }^{40-54} \mathrm{~A}$ study of 100 unrelated patients with idiopathic $D C^{41}$ identified desmosomal gene sequence variants (some previously associated with ARVC) in 18 patients, five of which were classified as pathogenic. In limited co-segregation studies in two of the pedigrees, no mutant gene carriers fulfilled diagnostic criteria for either ARVC or DCM, but frequent ventricular ectopy and/or myopathic patterns of late gadolinium enhancement (LGE) were detected in some mutation carriers with normal echocardiograms. These findings illustrate the frequency of genetic variants in patients with DCM and the heterogeneous and often subtle manifestations of disease in relatives who carry the same variant.

\section{Advanced cardiac imaging and myocardial characterisation}

Tissue characterisation, CMR's unique contribution to non-invasive imaging, can differentiate normal myocardium from oedematous, fibrotic and infiltrated myocardium and can detect fatty change. In specific clinical situations, the profile, spatial distribution and temporal characteristics of tissue abnormalities, can differentiate between causes of cardiac damage. ${ }^{55-57}$ Myocardial LGE after myocardial infarction is typically subendocardial; in non-ischaemic DCM, LGE is detected in at least onethird of cases $^{58-60}$ and is typically mid-wall or subepicardial, but neither the presence of LGE or its localisation are specific for any particular aetiology. ${ }^{416162}$ However, recently published work suggests that the presence of LGE may be a marker of disease severity and is prognostically important in some settings. ${ }^{58-60} 63-65$ Management strategies that incorporate LGE findings have not been formulated or assessed.

CMR in the diagnosis of myocarditis, in particular with respect to acute and chronic abnormalities in tissue characteristics and their relation to disease progression to DCM and to the development of heart failure. ${ }^{565766}$

Larger studies are now needed to validate CMR diagnostic criteria for myocarditis in a variety of clinical settings (eg, in a cohort with idiopathic DCM), and to establish whether CMR findings have prognostic value. 


\section{Treatment}

Guidelines for pharmacological and device therapies in heart failure make little reference to aetiology-specific management. In considering indications for device therapy (both ICD and cardiac resynchronisation therapy), major national guidelines suggest slightly different criteria for non-ischaemic than for ischaemic heart failure that recognise the greater likelihood that, on average, nonischaemic DCM may have a better prognosis. ${ }^{67-69}$

Recent work by Millat et al identifies some of the hazards in assuming homogeneity among patients with DCM. ${ }^{45}$ They demonstrated that nearly $10 \%$ of unrelated

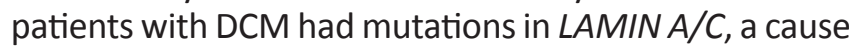
of DCM associated with particularly high risks of ventricular arrhythmias and progressive conduction disease. ${ }^{70}$ Similar complications are also common in the cardiomyopathy associated with myotonic dystrophy ${ }^{7172}$

More conventional imaging findings may also be important in refining device therapy. ${ }^{73-75}$ Recent publications describe the prognostic importance of functional mitral regurgitation, a feature of DCM related to LV geometry, contractility and dyssynchrony. ${ }^{76} 77$ Rossi et al demonstrate, in non-ischaemic DCM, that functional mitral regurgitation is associated with doubling of a combined end point of all-cause mortality hospitalisation and worsening heart failure. ${ }^{74}$

\section{Genetics}

Many of the inherited cardiac conditions for which a genetic cause was first detected were highly penetrant monogenic diseases with a readily detectable cardiac phenotype amenable to discovery approaches such as genetic linkage. In suitably sized pedigrees with highly penetrant disease, linkage analysis can provide statistical associations between a genetic locus/mutation with a disease phenotype (ie, a statistically robust co-segregation expressed as a logarithmic or LOD score). This classic genetic technique continues to deliver new genetic findings in hereditary heart muscle disease. ${ }^{7879}$

More restricted candidate approaches in mutation detection have predominated in recent research; advantages include lower costs and an ability to study small families or individuals with low penetrance disease. Candidate genes are selected for a number of reasons that include membership in a gene group already associated with disease (eg, sarcomeric or desmosomal genes), an understanding that the gene's function may be important in the development of the phenotype (eg, genes involved in hypertrophic signalling) and particular features identified in the disease state (eg, differences in myocardial gene expression profiles between affected and normal subjects).

Recently published work presenting associations between new candidate genes and heart muscle disease may be considered in at least three categories: descriptions of relative frequencies of genetic abnormalities in genes known to cause a specific disease ${ }^{18434548}$; searches for sequence variants in genes associated with one heart muscle disease in another (cross-over or overlapping phenotypes) $28414246-495280$ and discovery of se- quence variants in new candidates not previously associated with any cardiac disease. ${ }^{81} 82$

Until recently, largely empirical evidence has been used to support candidate gene choice but selection methods influence the pre-test probability that variants in the target gene are disease causing. More quantitative techniques for the identification of candidate genes have recently been described. ${ }^{83-85}$

Villard and colleagues performed genome-wide association studies on pooled DNA samples obtained from patients with apparently sporadic idiopathic DCM. ${ }^{83}$ Two single nuclear polymorphisms on chromosomes 1 p36 and $10 q 26$ were significantly associated with DCM. An interpretation of these data is that these loci contain genes that play an important, but not causal, role in the development of sporadic DCM. However, several mutations in one of the candidate genes (BAG3, chromosome 10 locus) were identified in patients with familial DCM; several of these mutations co-segregated with the familial phenotype. It must be noted that it was unlikely that this study was designed to identify candidate genetic causes of DCM; in any case, none of the loci containing any of the many known DCM genes were identified.

Remarkably, another methodological first for cardiomyopathy also identified BAG3 as a cause of DCM. In the proband and in three affected family members of a multigenerational pedigree with autosomal dominant DCM, Norton et al performed both whole exon sequencing and a genome-wide assessment of copy number variation (CNV). ${ }^{84}$ After exome sequencing, none of the genetic variants identified in the proband co-segregated with the disease. The high-density genome-wide CNV assay (said to have single-exon resolution) detected a deletion encompassing exon 4 of BAG3 that co-segregated with disease. As mutation detection strategies reliant only on sequencing coding regions of the genome will fail to detect CNVs caused by large deletions or insertions, future CNV studies may yet tell us that large deletions in genes already associated with cardiomyopathies are an important cause of these diseases.

Finally, using a more basic and inclusive approach to identifying candidate genes, Neely and colleagues assessed the effects of cardiac-specific 'knock-down' (with RNA-i) of more than 7000 genes in Drosophila reared under conditions of cardiac stress. ${ }^{85}$ The authors identified nearly 500 evolutionarily conserved genes and pathways likely to have critical and conserved roles in the cardiovascular system. Their findings identify many targets for future candidate gene studies; for example, altered cardiac repolarisation is associated with a common polymorphism in the human isoform of a gene associated with a DCM phenotype in Drosophila.

\section{New technology}

The term next-generation sequencing (NGS) refers to a number of technologies that provide massively parallel, high throughput DNA sequencing. Technological advances in the preparation of DNA before sequencing (enriching and labelling, for example), in sequencing 
chemistry and in bioinformatics will result in reduced costs and improvements in automation, accuracy and coverage. Recently published reviews describe NGS in more detail, and specifically with reference to inherited heart disease. ${ }^{8687}$

As the inherited heart muscle diseases have striking allelic and locus heterogeneity (multiple mutations in many different genes), NGS will enable a step-change in both research and diagnostic applications of DNA sequencing. The first few reports of NGS being used to detect mutations in these conditions were published in 2010 and 2011. ${ }^{8488-90}$

\section{Summary}

As we approach the end of 2011 it is clear that the next few years are going to be dominated by the application of new high throughput genetic screening techniques, capable of screening the entire exome or indeed genome. Understanding the data generated by these techniques will require new and equally sophisticated analysis of large and complex datasets, using a systems biology approach with deeper phenotyping and advanced modelling techniques that have the flexibility for continuous update, refinement with discovery of new knowledge. Exciting new developments that may also transform cardiomyopathy research include those of infrastructure and organisation (multi-centre collaborations) and spin-offs from the field of regenerative medicine research. For clinical researchers that translate this information to the clinic the focus will however remain the same; namely improvement of quality and quantity of life.

\section{References}

1 Hagege AA, Caudron E, Damy T, et al. Screening patients with hypertrophic cardiomyopathy for fabry disease using a filterpaper test: the focus study. Heart 2011;97:131-6.

2 Olivotto I, Maron BJ, Appelbaum E, et al. Spectrum and clinical significance of systolic function and myocardial fibrosis assessed by cardiovascular magnetic resonance in hypertrophic cardiomyopathy. Am J Cardiol 2010;106:261-7.

3 O'Hanlon R, Grasso A, Roughton M, et al. Prognostic significance of myocardial fibrosis in hypertrophic cardiomyopathy. J Am Coll Cardiol 2010;56:867-74.

4 Bruder O, Wagner A, Jensen CJ, et al. Myocardial scar visualized by cardiovascular magnetic resonance imaging predicts major adverse events in patients with hypertrophic cardiomyopathy. J Am Coll Cardiol 2010;56:875-87.

5 Flett AS, Hayward MP, Ashworth MT, et al. Equilibrium contrast cardiovascular magnetic resonance for the measurement of diffuse myocardial fibrosis: preliminary validation in humans. Circulation 2010;122:138-44.

6 Ho CY, Lopez B, Coelho-Filho OR, et al. Myocardial fibrosis as an early manifestation of hypertrophic cardiomyopathy. N Engl J Med 2010;363:552-63.

7 Ingles J, McGaughran J, Scuffham PA, et al. A cost-effectiveness model of genetic testing for the evaluation of families with hypertrophic cardiomyopathy. Heart 2011. (in press). doi:10.1136/ heartjnl-2011-300368

8 Wordsworth S, Leal J, Blair E, et al. DNA testing for hypertrophic cardiomyopathy: a cost-effectiveness model. Eur Heart J 2010;31:926-35.

9 Agarwal S, Tuzcu EM, Desai MY, et al. Updated meta-analysis of septal alcohol ablation versus myectomy for hypertrophic cardiomyopathy. J Am Coll Cardiol 2010;55:823-34.
10 Ten Cate FJ, Soliman OI, Michels M, et al. Long-term outcome of alcohol septal ablation in patients with obstructive hypertrophic cardiomyopathy: a word of caution. Circ Heart Fail 2010;3:362-9.

11 Nishimura RA, Ommen SR. Septal reduction therapy for obstructive hypertrophic cardiomyopathy and sudden death: what statistics cannot tell you. Circ Cardiovasc Interv 2010;3:91-3.

12 Leonardi RA, Kransdorf EP, Simel DL, et al. Meta-analyses of septal reduction therapies for obstructive hypertrophic cardiomyopathy: comparative rates of overall mortality and sudden cardiac death after treatment. Circ Cardiovasc Interv 2010;3:97-104.

13 Lawrenz T, Borchert B, Leuner C, et al. Endocardial radiofrequency ablation for hypertrophic obstructive cardiomyopathy: acute results and 6 months' follow-up in 19 patients. J Am Coll Cardiol 2011;57:572-6.

14 Galve E, Sambola A, Saldana G, et al. Late benefits of dual-chamber pacing in obstructive hypertrophic cardiomyopathy: a 10-year follow-up study. Heart 2010;96:352-6.

15 Mohiddin SA, Page SP. Long-term benefits of pacing in obstructive hypertrophic cardiomyopathy. Heart 2010;96:328-30.

16 Bos JM, Maron BJ, Ackerman MJ, et al. Role of family history of sudden death in risk stratification and prevention of sudden death with implantable defibrillators in hypertrophic cardiomyopathy. Am J Cardiol 2010;106:1481-6.

17 Christiaans I, van Engelen K, van Langen IM, et al. Risk stratification for sudden cardiac death in hypertrophic cardiomyopathy: systematic review of clinical risk markers. Europace 2010;12:313-21.

18 CO'Mahony, PD Lambiase, G Quarta, et al. The long-term survival and the risks and benefits of implantable cardioverter defibrillators in patients with hypertrophic cardiomyopathy. Heart. Published Online First: 13 July 2011. doi:10.1136/hrt.2010.217182

19 Kaski JP, Syrris P, Esteban MT, et al. Prevalence of sarcomere protein gene mutations in preadolescent children with hypertrophic cardiomyopathy. Circ Cardiovas Genet 2009;2:436-41.

20 JP Moak, ES Leifer, D Tripodi, et al. Long-term follow-up of children and adolescents diagnosed with hypertrophic cardiomyopathy: risk factors for adverse arrhythmic events. Pediatr Cardiol. Published Online First: 13 April 2011. PMID:21487794

21 Gao D, Ning N, Niu X, et al. Trimetazidine: a meta-analysis of randomised controlled trials in heart failure. Heart 2011;97:278-86.

22 Abozguia K, Elliott P, McKenna W, et al. Metabolic modulator perhexiline corrects energy deficiency and improves exercise capacity in symptomatic hypertrophic cardiomyopathy. Circulation 2010;122:1562-9.

23 Corrado D, Basso C, Pilichou K, et al. Molecular biology and clinical management of arrhythmogenic right ventricular cardiomyopathy/dysplasia. Heart 2011;97:530-9.

24 Marcus FI, McKenna WJ, Sherrill D, et al. Diagnosis of arrhythmogenic right ventricular cardiomyopathy/dysplasia: proposed modification of the task force criteria. Eur Heart J 2010;31:806-14.

25 Fressart V, Duthoit G, Donal E, et al. Desmosomal gene analysis in arrhythmogenic right ventricular dysplasia/cardiomyopathy: spectrum of mutations and clinical impact in practice. Europace 2010;12:861-8.

26 Otten E, Asimaki A, Maass A, et al. Desmin mutations as a cause of right ventricular heart failure affect the intercalated disks. Heart Rhythm 2010;7:1058-64. the official journal of the Heart Rhythm Society.

27 Klauke B, Kossmann S, Gaertner A, et al. De novo desmin-mutation $\mathrm{n} 116 \mathrm{~s}$ is associated with arrhythmogenic right ventricular cardiomyopathy. Hum Mol Genet 2010;19:4595-607.

28 Taylor M, Graw S, Sinagra G, et al. Genetic variation in titin in arrhythmogenic right ventricular cardiomyopathy-overlap syndromes. Circulation 2011;124:876-85.

29 Kapplinger JD, Landstrom AP, Salisbury BA, et al. Distinguishing arrhythmogenic right ventricular cardiomyopathy/dysplasiaassociated mutations from background genetic noise. J Am Coll Cardiol 2011;57:2317-27.

30 Cox MG, van der Zwaag PA, van der Werf C, et al. Arrhythmogenic right ventricular dysplasia/cardiomyopathy: pathogenic 
desmosome mutations in index-patients predict outcome of family screening: Dutch arrhythmogenic right ventricular dysplasia/cardiomyopathy genotype-phenotype follow-up study. Circulation 2011;123:2690-700.

31 Quarta G, Muir A, Pantazis A, et al. Familial evaluation in arrhythmogenic right ventricular cardiomyopathy: impact of genetics and revised task force criteria. Circulation 2011;123:2701-9.

32 Protonotarios N, Anastasakis A, Antoniades L, et al. Arrhythmogenic right ventricular cardiomyopathy/dysplasia on the basis of the revised diagnostic criteria in affected families with desmosomal mutations. Eur Heart J 2011;32:1097-104.

33 La Gerche A, Robberecht C, Kuiperi C, et al. Lower than expected desmosomal gene mutation prevalence in endurance athletes with complex ventricular arrhythmias of right ventricular origin. Heart 2010;96:1268-74.

34 Quarta G, Ward D, Tome Esteban MT, et al. Dynamic electrocardiographic changes in patients with arrhythmogenic right ventricular cardiomyopathy. Heart 2010;96:516-22.

35 Corrado D, Calkins H, Link MS, et al. Prophylactic implantable defibrillator in patients with arrhythmogenic right ventricular cardiomyopathy/dysplasia and no prior ventricular fibrillation or sus tained ventricular tachycardia. Circulation 2010;122:1144-52.

36 Wiltshire SA, Leiva-Torres GA, Vidal SM. Quantitative trait locus analysis, pathway analysis, and consomic mapping show genetic variants of tnni3k, fpgt, or h28 control susceptibility to viral myocarditis. J Immunol 2011;186:6398-405.

37 Gorbea C, Makar KA, Pauschinger M, et al. A role for toll-like receptor 3 variants in host susceptibility to enteroviral myocarditis and dilated cardiomyopathy. J Biol Chem 2010;285:23208-23.

38 Horne BD, Rasmusson KD, Alharethi R, et al. Genome-wide significance and replication of the chromosome 12 p11.22 locus near the pthlh gene for peripartum cardiomyopathy. Circ Cardiovasc Genet 2011;4:359-66.

39 van Spaendonck-Zwarts KY, van Tintelen JP, van Veldhuisen DJ, et al. Peripartum cardiomyopathy as a part of familial dilated cardiomyopathy. Circulation 2010;121:2169-75.

40 Hoedemaekers YM, Caliskan K, Michels M, et al. The importance of genetic counseling, DNA diagnostics, and cardiologic family screening in left ventricular noncompaction cardiomyopathy. Circ Cardiovasc Genet 2010;3:232-9.

41 Elliott P, O'Mahony C, Syrris P, et al. Prevalence of desmosomal protein gene mutations in patients with dilated cardiomyopathy. Circ Cardiovasc Genet 2010;3:314-22.

42 Garcia-Pavia P, Syrris P, Salas C, et al. Desmosomal protein gene mutations in patients with idiopathic dilated cardiomyopathy undergoing cardiac transplantation: a clinicopathological study. Heart 2011;97:1744-52.

43 Cowan J, Li D, Gonzalez-Quintana J, et al. Morphological analysis of 13 Imna variants identified in a cohort of 324 unrelated patients with idiopathic or familial dilated cardiomyopathy. Circ Cardiovasc Genet 2010;3:6-14.

44 Caleshu C, Day S, Rehm HL, et al. Use and interpretation of genetic tests in cardiovascular genetics. Heart 2010;96:1669-75.

45 Millat G, Bouvagnet $P$, Chevalier $P$, et al. Clinical and mutational spectrum in a cohort of 105 unrelated patients with dilated cardiomyopathy. Eur J Med Genet 2011;54:e570-5.

46 Moller DV, Andersen PS, Hedley P, et al. The role of sarcomere gene mutations in patients with idiopathic dilated cardiomyopathy. Eur J Hum Genet 2009;17:1241-9.

47 Probst S, Oechslin E, Schuler P, et al. Sarcomere gene mutations in isolated left ventricular noncompaction cardiomyopathy do not predict clinical phenotype. Circ Cardiovasc Genet 2011;4:367-74.

48 Rampersaud E, Siegfried JD, Norton N, et al. Rare variant mutations identified in pediatric patients with dilated cardiomyopathy. Prog Pediatr Cardiol 2011;31:39-47.

49 Caleshu C, Sakhuja R, Nussbaum RL, et al. Furthering the link between the sarcomere and primary cardiomyopathies: restrictive cardiomyopathy associated with multiple mutations in genes previously associated with hypertrophic or dilated cardiomyopathy. Am J Med Genet A 2011;155A:2229-35.

50 Kaski JP, Syrris P, Burch M, et al. Idiopathic restrictive cardiomyopathy in children is caused by mutations in cardiac sarcomere protein genes. Heart 2008;94:1478-84.
51 Girolami F, Ho CY, Semsarian C, et al. Clinical features and outcome of hypertrophic cardiomyopathy associated with triple sarcomere protein gene mutations. J Am Coll Cardiol 2010;55:1444-53.

52 Hershberger RE, Norton N, Morales A, et al. Coding sequence rare variants identified in mybpc3, myh6, tpm 1 , tnnc1, and tnni3 from 312 patients with familial or idiopathic dilated cardiomyopathy. Circ Cardiovasc Genet 2010;3:155-61.

53 Rampersaud E, Kinnamon DD, Hamilton K, et al. Common susceptibility variants examined for association with dilated cardiomyopathy. Ann Hum Genet 2010;74:110-16.

54 Armel TZ, Leinwand LA. Mutations at the same amino acid in myosin that cause either skeletal or cardiac myopathy have distinct molecular phenotypes. J Mol Cell Cardiol 2010;48:1007-13.

55 Friedrich MG, Sechtem U, Schulz-Menger J, et al. Cardiovascular magnetic resonance in myocarditis: a jacc white paper. J Am Coll Cardiol 2009;53:1475-87.

56 Monney PA, Sekhri N, Burchell T, et al. Acute myocarditis presenting as acute coronary syndrome: role of early cardiac magnetic resonance in its diagnosis. Heart 2010;97:1312-18.

57 Leurent G, Langella B, Fougerou C, et al. Diagnostic contributions of cardiac magnetic resonance imaging in patients presenting with elevated troponin, acute chest pain syndrome and unobstructed coronary arteries. Arch Cardiovasc Dis 2011;104:161-70.

58 Assomull RG, Prasad SK, Lyne J, et al. Cardiovascular magnetic resonance, fibrosis, and prognosis in dilated cardiomyopathy. J Am Coll Cardiol 2006;48:1977-85.

59 Lehrke S, Lossnitzer D, Schob M, et al. Use of cardiovascular magnetic resonance for risk stratification in chronic heart failure: prognostic value of late gadolinium enhancement in patients with non-ischaemic dilated cardiomyopathy. Heart 2011;97:727-32.

60 Malaty AN, Shah DJ, Abdelkarim AR, et al. Relation of replacement fibrosis to left ventricular diastolic function in patients with dilated cardiomyopathy. J Am Soc Echocardiogr 2011;24:333-8. official publication of the American Society of Echocardiography.

61 Holmstrom M, Kivisto S, Helio T, et al. Late gadolinium enhanced cardiovascular magnetic resonance of lamin a/c gene mutation related dilated cardiomyopathy. J Cardiovasc Magn Reson 2011;13:30. official journal of the Society for Cardiovascular Magnetic Resonance.

62 Nucifora G, Aquaro GD, Pingitore A, et al. Myocardial fibrosis in isolated left ventricular non-compaction and its relation to disease severity. Eur J Heart Fail 2011;13:170-6.

63 Alter $\mathrm{P}, \mathrm{Rupp} \mathrm{H}$, Adams $\mathrm{P}$, et al. Occurrence of late gadolinium enhancement is associated with increased left ventricular wall stress and mass in patients with non-ischaemic dilated cardiomyopathy. Eur J Heart Fail 2011;13:937-44.

64 Wu KC, Weiss RG, Thiemann DR, et al. Late gadolinium enhancement by cardiovascular magnetic resonance heralds an adverse prognosis in nonischemic cardiomyopathy. J Am Coll Cardiol 2008;51:2414-21.

65 Tigen K, Karaahmet T, Kirma C, et al. Diffuse late gadolinium enhancement by cardiovascular magnetic resonance predicts significant intraventricular systolic dyssynchrony in patients with non-ischemic dilated cardiomyopathy. J Am Soc Echocardiogr 2010;23:416-22. official publication of the American Society of Echocardiography.

66 Escher F, Westermann D, Gaub R, et al. Development of diastolic heart failure in a 6-year follow-up study in patients after acute myocarditis. Heart 2011;97:709-14.

67 Dickstein K, Vardas PE, Auricchio A, et al. 2010 focused update of esc guidelines on device therapy in heart failure: an update of the 2008 esc guidelines for the diagnosis and treatment of acute and chronic heart failure and the 2007 esc guidelines for cardiac and resynchronization therapy. Developed with the special contribution of the heart failure association and the european heart rhythm association. Eur Heart J 2010;31:2677-87.

68 McNamara DM, Starling RC, Cooper LT, et al. Clinical and demographic predictors of outcomes in recent onset dilated cardiomyopathy results of the IMAC (intervention in myocarditis and acute cardiomyopathy)-2 study. J Am Coll Cardiol 2011;58:1112-18.

69 Verma A, Wulffhart Z, Lakkireddy D, et al. Incidence of left ventricular function improvement after primary prevention icd im- 
plantation for non-ischaemic dilated cardiomyopathy: a multicentre experience. Heart 2010;96:510-15.

70 Meune C, Van Berlo JH, Anselme F, et al. Primary prevention of sudden death in patients with lamin a/c gene mutations. N Engl J Med 2006;354:209-10.

71 McNally EM, Sparano D. Mechanisms and management of the heart in myotonic dystrophy. Heart 2011;97:1094-100.

72 Lallemand B, Clementy N, Brunet AB, et al. The evolution of infrahissian conduction time in myotonic dystrophy patients: clinical implications. Heart 2011. (in press). doi: 10.1136/heartjnl-2011-300143

73 van Bommel RJ, Marsan NA, Delgado V, et al. Cardiac resynchronization therapy as a therapeutic option in patients with moderate-severe functional mitral regurgitation and high operative risk. Circulation 2011;124:912-19.

74 Rossi A, Dini FL, Faggiano P, et al. Independent prognostic value of functional mitral regurgitation in patients with heart failure. A quantitative analysis of 1256 patients with ischaemic and nonischaemic dilated cardiomyopathy. Heart 2011;97:1675-80.

75 Bertini M, Ziacchi M, Biffi M, et al. Effects of cardiac resynchronisation therapy on dilated cardiomyopathy with isolated ventricular non-compaction. Heart 2011;97:295-300.

76 Agricola E, Oppizzi M, Galderisi M, et al. Role of regional mechanical dyssynchrony as a determinant of functional mitral regurgitation in patients with left ventricular systolic dysfunction. Heart 2006;92:1390-5.

77 Donal E, De Place C, Kervio G, et al. Mitral regurgitation in dilated cardiomyopathy: Value of both regional left ventricular contractility and dyssynchrony. Eur J Echocardiogr 2009;10:133-8. the journal of the Working Group on Echocardiography of the European Society of Cardiology.

78 Chiu C, Bagnall RD, Ingles J, et al. Mutations in alpha-actinin-2 cause hypertrophic cardiomyopathy: a genome-wide analysis. J Am Coll Cardiol 2010;55:1127-35.

79 Brauch KM, Karst ML, Herron KJ, et al. Mutations in ribonucleic acid binding protein gene cause familial dilated cardiomyopathy. J Am Coll Cardiol 2009;54:930-41.

80 Morales A, Painter T, Li R, et al. Rare variant mutations in pregnancy-associated or peripartum cardiomyopathy. Circulation 2010;121:2176-82.
81 Arimura T, Bos JM, Sato A, et al. Cardiac ankyrin repeat protein gene (ankrd1) mutations in hypertrophic cardiomyopathy. J Am Coll Cardiol 2009;54:334-42.

82 Moulik M, Vatta M, Witt SH, et al. Ankrd1, the gene encoding cardiac ankyrin repeat protein, is a novel dilated cardiomyopathy gene. J Am Coll Cardiol 2009;54:325-33.

83 Villard E, Perret C, Gary F, et al. A genome-wide association study identifies two loci associated with heart failure due to dilated cardiomyopathy. Eur Heart J 2011;32:1065-76.

84 Norton N, Li D, Rieder MJ, et al. Genome-wide studies of copy number variation and exome sequencing identify rare variants in bag3 as a cause of dilated cardiomyopathy. Am J Hum Genet 2011;88:273-82.

85 Neely GG, Kuba K, Cammarato A, et al. A global in vivo drosophila rnai screen identifies not3 as a conserved regulator of heart function. Cell 2010;141:142-53.

86 Voelkerding KV, Dames S, Durtschi JD. Next generation sequencing for clinical diagnostics-principles and application to targeted resequencing for hypertrophic cardiomyopathy: a paper from the 2009 william beaumont hospital symposium on molecular pathology. J Mol Diagn 2010;12:539-51.

87 Ware JS, Roberts AM, Cook SA. Next generation sequencing for clinical diagnostics and personalised medicine: implications for the next generation cardiologist. Heart 2011. (in press). doi: 10.1136/heartjnl-2011-300742

88 Dames S, Durtschi J, Geiersbach K, et al. Comparison of the illumina genome analyzer and roche $454 \mathrm{gs}$ flx for resequencing of hypertrophic cardiomyopathy-associated genes. J Biomol Tech 2010;21:73-80.

89 Meder B, Haas J, Keller A, et al. Targeted next-generation sequencing for the molecular genetic diagnostics of cardiomyopathies. Circ Cardiovasc Genet 2011;4:110-22.

90 Gowrisankar S, Lerner-Ellis JP, Cox S, et al. Evaluation of secondgeneration sequencing of 19 dilated cardiomyopathy genes for clinical applications. J Mol Diagnost 2010;12:818-27. 